http://jmscr.igmpublication.org/home/ ISSN (e)-2347-176x ISSN (p) 2455-0450

crossref DOI: https://dx.doi.org/10.18535/jmscr/v9i4.26

Journal Of Medical Science And Clinical Research

\title{
A Clinical study on Ophthalmic Manifestation of Rheumatoid Arthritis based on patients attending Rheumatology Department of Medical College Hospital, Thiruvananthapuram, South Kerala
}

\author{
Authors \\ S. Rema Devi ${ }^{1}$, Jacob Antony ${ }^{2}$ \\ ${ }^{1}$ Assistant Professor, Regional Institute of Ophthalmology, Government Medical College, \\ Thiruvananthapuram, Kerala 695 035, India \\ ${ }^{2}$ Associate Professor, Department of Rheumatology, Government Medical College, Thiruvananthapuram,
} Kerala 695 011, India

\begin{abstract}
Background: Rheumatoid Arthritis (RA) is an auto Immune chronic Inflammatory disorder of worldwide distribution and it causes painful and destructing articular conditions. Extra articular manifestation in RA are more common in sero +ve patients. The extra articular manifestations includes, epicarditis, pleuritis, major cutaneous, vasculitis, ophthalmic manifestations, glomerulo nephritis, and other type of vasculitis. Ophthalmic manifestation caused by RA causes severe ocular morbidities if not screened at an early stage.

Materials and Methods: A hospital based prospective observational study on patient attended at Rheumatology Department of Medical College, Thiruvananthapuram who have diagnosed as Rheumatoid arthritis were examined and called for detailed ocular evaluation at RIO Clinic. A total of 198 cases were screened during August 2019 to January 2021. All patients were subjected to detailed history, pertaining to age, duration of the disease systemic manifestation of RA, drug history and its duration and symptoms related to ophthalmic manifestation were collected. Protocol based treatment were offered after detailed ophthalmic evaluations tests and effect of treatment was studied during follow up periods. Data of the patients were recorded on a pre-fabricated proforma. Data was statistically analysed by chi-square analysis odds ratio and Logistic regression analysis.

Results: 115 (58.08\%) patients of RA (Rheumatoid arthritis) showed Ophthalmic manifestations KCS. (dry eye manifestations) was the most common complications which responds well to artificial tear substitutes on daily application. Complications like episcleritis scleritis and iridocyclitis and keratitis were noted out and active emergency strategies were given to avoid ophthalmic morbidities and this helped to alleviate the problems of severe Vn Impairment. Drugs related to ocular complications like quinolen toxicity and posterior sub capsular opacity due to steroid toxicity were ruled out and strict follow up measures were given along with the guidance of the Rheumatologist.

Conclusion

1. Rheumatoid arthritis is also a systemic auto immune disease have signs and symptoms of the eye.

2. KCS (Dry Eye manifestation) were the commonest complication.

3. Early Ophthalmolgic interference and active treatment regimen can reduce further extent of ocular morbidities and thus Vn threatening conditions.

Keywords: Rheumatoid arthritis, peripheral Ulcerative keratitis, hydroxy- chloroquine, kerato conjunctivitis sicca, Non steroidal anti inflammatory drugs, posterior sub capsular cataract.
\end{abstract}




\section{Background}

RA is a common chronic systemic Inflammatory disorders of unknown etiology that primarily involve joints. Approximately more than $25 \%$ of patients with RA will have ocular manifestation. Hence in the present study indicated the urgent need of the RA patients for ophthalmic Screening and management to avoid Vision impairment and severe sight threatening conditions.

RA confirmed cases were studied at RIO (Regional Institute of Ophthalmology, Thiruvananthapuram) prospectively and the data obtained were statistically analysed commonly noted ophthalmic manifestation are:-

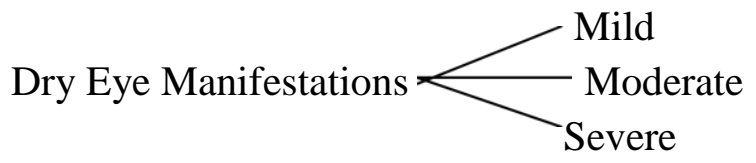

Episcleritis, scleritis, scleritis with PUK, Iridocyclilis, Quinolen maculopathy and posterior subcapsular opacity (Posterior-subcapsular Cataract) in relation with systemic therapy related complications.

DRY eye manifestations were the commonest. Adequate treatment measure were offered to those patients who have showed abnormal Schirmer test and diminished corneal tear meniscus. Among the episcleritis, the simple diffuse type was noted commonly. Scleritis was mainly of the diffuse anterior type in which ocular surface edema and chemosis was noted only in doubtful cases phenylephrine test with $2 \%$ drops are used for differentiation between episcleritis and scleritis patients. Most of the scleritis patients were treated with oral and topical steroids and NSAIDS. Nodular scleritis was noted in few cases with complaints of intense pain on palpation. Yet with starting of intense therapy had brought change in reduction of symptoms like pain and redness. Topical steroid and NSAID resistant cases were responds well to immuno suppressive like cyclosporine drops.

Pheripheral Ulcerative Keratitis was noted in five cases.
Treatment modality included were topical preparation of $1 \%$ medroxyprogesterone or $20 \%$ $\mathrm{N}$, acetylcysteine along with lubricating gel, antibiotic drops, or ointment, and cycloplegic drops in favour of aiding re-epithelization. Anterior, uveitis patients were treated with protocol based drugs and were responded to treatment very effectively. Many patients referred from Rheumatology Department for evaluation of quinoline and steroid induced toxicity. Some patients were using quinoline for more than five years without any follow up or ophthalmic evaluations while on screening they were presented with drop in vision acuity and scotomas on macular function tests. Hence urgent regular ophthalmic follow up evaluations and monitoring was suggested to these patients. Some patients receiving systemic steroids were having postering subcapsular cataracts were presented with decreased vision on both eye on Vn acuity screening tests. Hence to these patients also we had given instructions like an urgent need of attending the Rheumatology clinic on regular intervals to receive new suggestions of any change in the prescribed dose schedule of systemic drugs.

\section{Aim}

1. To find out the ophthalmic manifestation of patients with Rheumatoid arthritis attending a tertiary eye hospital in southern Kerala and their management.

2. Treatment outcomes of those patients who receiving treatment.

Settings: Regional Institute of Ophthalmology Government Medical College Hospital, Thiruvananthapuram, South Kerala.

\section{Materials and Methods}

The study was designed as a hospital based prospective observational study and patients presented at Rheumatology Department of Government Medical College hospital, Thiruvananthapuram, identified as RA were examined and called for detailed ocular evaluations at RIO. A total of 198 patients were 
screened during August 2019 to January 2021. After getting consent from the Institutional Human Ethical Committee all patients were subjected to detailed evaluation include history taking as to age, the duration of illness, drug history (history of treatment taking) and systemic illness and ocular symptoms were collected and subjected to detailed ocular examination was done using torch light and slit lamp, visual acuity test and detailed funds examination was done. Patients who is on plaquenil (HcQ) were subjected to macular functions tests like Amsler Grid test, colour Vn tests, field analysis and B Scan evaluations. DRY eye Evaluation tests was done in almost all the patients protocol based treatment was given to all patients with ophthalmic manifestations and effect of treatment was evaluated during the $1^{\text {st }}$ visit and later follow up periods. Data of the patients were recorded on a pre fabricated proforma. Data was statistically analysed by chi-square analysis, odds ratio and Logistic regression analysis.

\section{Results}

198 patients with Rheumatoid factor positivity were screened from August 2019 to January 2021. Out of 198 patients 115 patients were showed Ophthalmic manifestation Results are as given below.

\section{Age Distribution}

Age (Years) group Analysis of the population revealed majorly of the subjects were in the age group of $5^{\text {th }}$ decade ie between $51-60$ years (Table 1 ) followed by $6^{\text {th }}$ decade (61-70 years).

\begin{tabular}{|l|c|c|}
\hline$\%$ & Frequency & Age \\
\hline 10.10 & 20 & $21-30$ \\
\hline 15.65 & 31 & $31-40$ \\
\hline 16.66 & 33 & $41-50$ \\
\hline 20.20 & 40 & $61-70$ \\
\hline 3.53 & 7 & $71-80$ \\
\hline
\end{tabular}

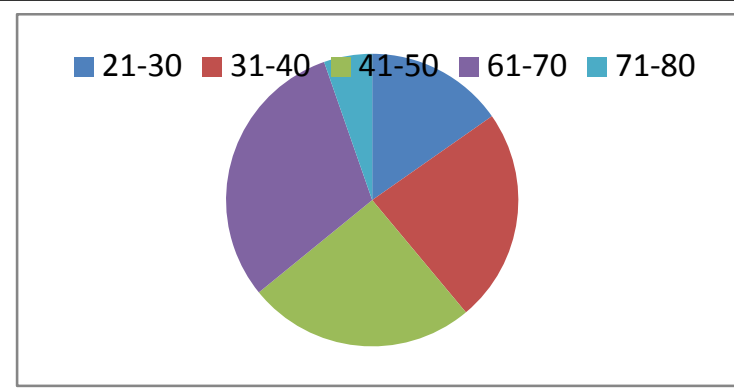

\section{Sex Distribution}

About the sex distribution (Table 2) majorly of the patients were females. $153(77.27 \%)$ and males were $45(22.72 \%)$. Female predominance was noted in this study.

\begin{tabular}{|l|c|c|}
\hline$\%$ & Number & Patients \\
\hline 22.72 & 45 & Males \\
\hline 77.27 & 153 & Females \\
\hline 99.99 & 198 & Total \\
\hline
\end{tabular}

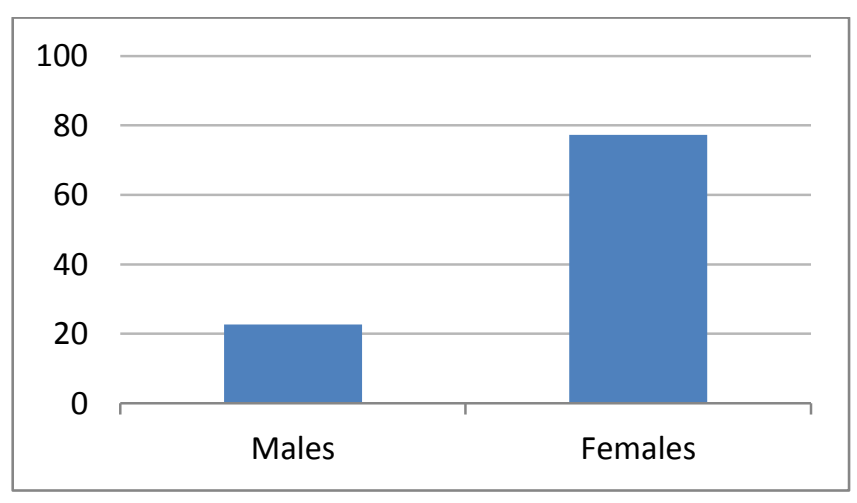

\section{Duration of Disease}

Majorly of the patients ie 118 in our study had RA for about $0-5$ years in the range followed by 6-10 yrs in 60 patients only. 20 patients had disease duration more than 10 years. Hence severe vision threatening conditions were not much in this study.

\begin{tabular}{|l|c|c|}
\hline$\%$ & Number & Duration (Years) \\
\hline 59.59 & 118 & $0-5$ \\
\hline 30.30 & 60 & $6-10$ \\
\hline 5.55 & 11 & $11-15$ \\
\hline 3.03 & 6 & $16-20$ \\
\hline 1.51 & 3 & $21-25$ \\
\hline & & \\
\hline
\end{tabular}




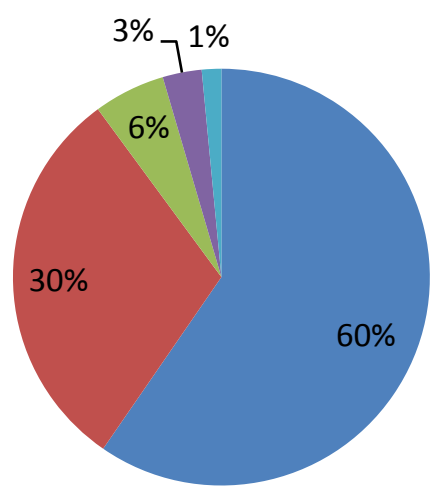

\section{Incidence of Ophthalmic Manifestation}

Results on ophthalmic manifestations were revealed as out of 198 patients 115 patients were showed. Hence Ophthalmic involvement was very high in this study.

\begin{tabular}{|l|c|c|}
\hline$\%$ & Number & Parameter \\
\hline 58.08 & 115 & $\begin{array}{c}\text { No. of Patients having } \\
\text { Ophthalmic Manifestation }\end{array}$ \\
\hline 41.91 & 83 & $\begin{array}{c}\text { No. of Patients not having } \\
\text { Ophthalmic Manifestation }\end{array}$ \\
\hline
\end{tabular}

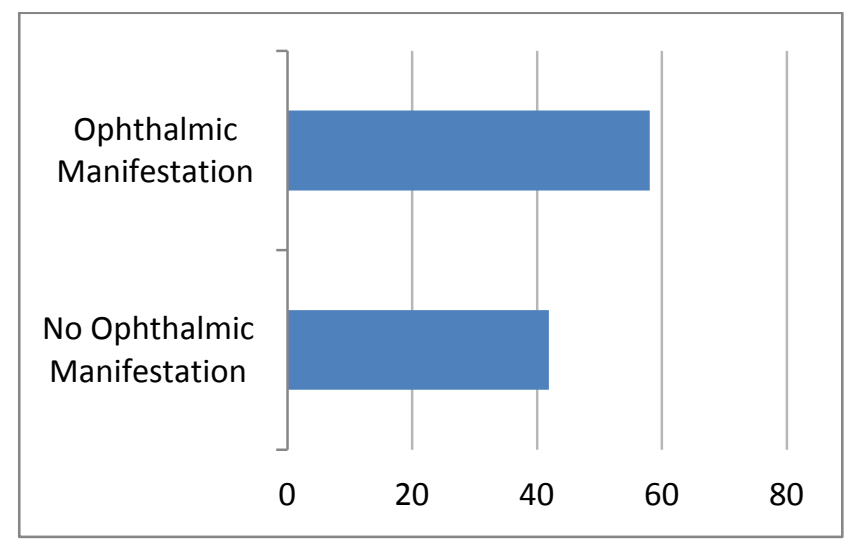

\section{Incidence of dry eye Manifestations}

Main manifestations detected were dry eyes (mild, moderate, severe) followed by episcleritis, scleritis, Iridocyclitis and peripheral Ulcerative keratitis.

\begin{tabular}{|l|c|c|}
\hline$\%$ & Frequency & Dry eye Manifestations \\
\hline & & Types \\
\hline 40.90 & 18 & Mild \\
\hline 36.36 & 16 & Moderate \\
\hline 22.72 & 10 & Severe \\
\hline 1.51 & 44 & Total \\
\hline
\end{tabular}

44 Patients showed different grades up dry eyes. Most of the patients were symptomatic. Those with severe dry eye were presented with Corneal punctuate erosions also. Diagnosis was made by schirmer test I and II and TBUT.

Ophthalmic Manifestation other than systemic therapy

\section{Opthalmic Manifestation Types}

\begin{tabular}{|l|c|c|}
\hline$\%$ & Number & Manifestation \\
\hline 52 & 26 & Episcleritis \\
\hline 24 & 12 & Scleritis \\
\hline 10 & 5 & Peripheral Ulcerative Keralitis \\
\hline 14 & 7 & Irido cyclilits \\
\hline 100 & 50 & Total \\
\hline
\end{tabular}

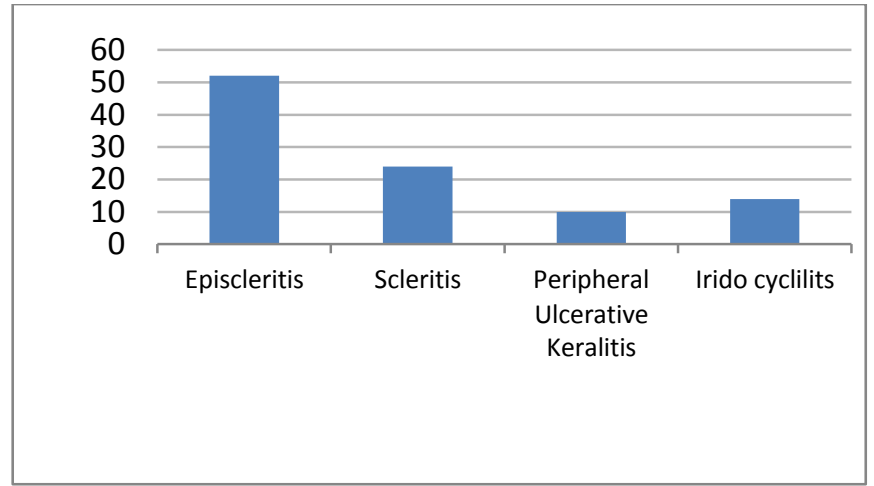

In this study Episcleritis was noted in 26 (52\%) patients. Scleritis as $12(24 \%)$ was mainly of the diffuse anterior type.

PUK were noted in 5 (10\%) patients only.

Anterior uveitis was noted in 7 (14\%) persons.

Systemic therapy related ophthal complications Systemic therapy related complication were noted in 11 persons. 9 persons were having PSC and 12 persons were having Quinolen (plquenil) maculopathy.

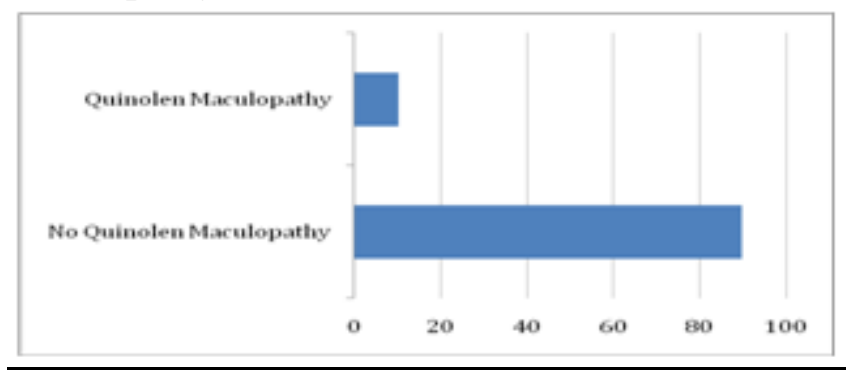

A. Quinolen Maculopathy

117 persons were using Hydroxychloroquine (Plaquenil) Out of which only 12 patients showed Quinolen Maculopathy (bulls eye) with lower 
corneal deposits they were having HCQ more than $5 \frac{1}{2}$ years.

\section{Complication due to Steroid}

Steroid toxicity in form of posterior sub capsular opacity were noted in 9 out of 115 . They were presented with symptoms like decreased, vision glare and decreased sensitivity to contrast.

\begin{tabular}{|c|c|c|c|c|}
\hline$\%$ & $\begin{array}{c}\text { No. of } \\
\text { patients } \\
\text { not having } \\
\text { PSC }\end{array}$ & $\%$ & $\begin{array}{c}\text { No. of } \\
\text { Patients } \\
\text { showed } \\
\text { PSC }\end{array}$ & $\begin{array}{c}\text { Total No. } \\
\text { of Patients } \\
\text { on Steriod }\end{array}$ \\
\hline 94.61 & 158 & 5.38 & 9 & 167 \\
\hline
\end{tabular}

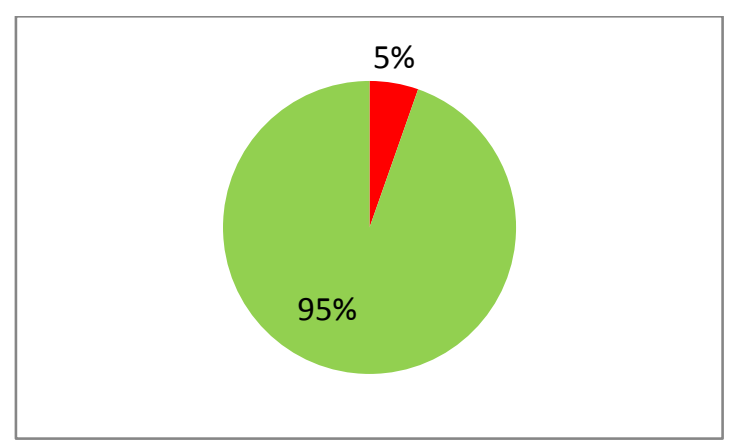

\section{Commonly Used Anti Rheumatoid Drug}

In this study commonly, used anti rheumatoid drugs prescribed from Rheumatology Department were noted as (HCQ) plaquenil, sulfasalazine, steroid (Methyl prednisolone) and methotrexate.

\begin{tabular}{|l|c|}
\hline No. of Patients & Drugs \\
\hline 117 & HCQ (Plaquenil) \\
\hline 90 & SULFASALAZINE \\
\hline 167 & STEROID \\
\hline 148 & Methotrexate \\
\hline
\end{tabular}

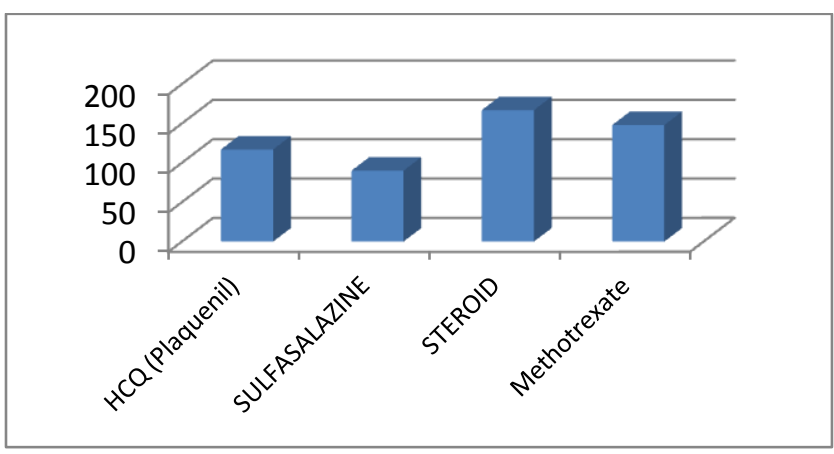

\section{Discussion}

RA has a worldwide distribution with an estimated prevalence of 1 to $2 \%$ a frequency that has been reported from several European and
North American Population (2002) ${ }^{1}$. The disease is also characterised in $80 \%$ of the patients by $+v e$ $\mathrm{RF}$ but there is also a group of auto - antibodies which are called anti-CCPS and are of great significance for the diagnosis of the disease (2002, $1998)^{2,3}$. Extra articular manifestations in RA are more common in sero $+{ }^{\mathrm{ve}}$ patients $(1973,1997)^{4,5}$. If $\mathrm{Rf}$ is present in high titre the higher the risk for more severe systemic disease $(1997)^{7}$. In this study also all Rf + vity patients were screened for ophthalmic manifestations RA is usually more common in women over the age of 55 Yrs with a peak incidence in the $5^{\text {th }}$ decade $(2015)^{6}$ as similar to this study. In this study population the duration of disease (RA) ranged between few months to 25 Yrs. $(60 \%)$ of the patients had disease duration in the range of 0-5 Yrs. a study similar to Wanchu et al $(1997)^{7}$. A Study conducted by S.Kalke etal $(2001)^{11}$ on 121 patients had disease duration in the range of 0-5 yrs. A study conducted by Reddy et al $(1997)^{8}$ shows that 13 out of 100 patients of RA (39\%) found to have eye involvement. The prevalence rate of ophthalmic manifestation in RA has been reported differently in different studies. The present study showed 115 (58\%) cases out of 198 patients.

Among the ophthalmic manifestations KCS (Dry Eyes) is the most common Ophthalmic association of RA. Rheumatoid process has been considered to be an aetiologically related to KCS by a study by Thampson and Eadie ${ }^{29}$. A Study by Moss etal $(2000)^{9}$ also showed $14.4 \%$ of the patient had dry eyes. Dry eye is caused by infiltration of the Lacrimal gland by $\mathrm{T}$ and $\mathrm{B}$ lymphocytes leads to secondary atrophy of the gland which is responsible for the further decrease of tears ${ }^{5,10}$ (1997, 1995).

The patient complaints of burning sensation of the eye, pain and blurred vision, mucus discharge and crusts are not uncommon so the dominant findings of KCs are two: decreased corneal tear meniscus and abnormal Schirmer Test (1993) ${ }^{12}$. KCS may progress to Stromal Ulceration and Corneal perforation $(2008)^{13}$. Females had more incidence than males. 
Schirmer Test is performed by putting a strip of Whatman's filter Paper No.41, 35 mm long, $5 \mathrm{~mm}$ wide is hooked over the lower eye lid. Such that $5 \mathrm{~mm}$ is bent into the lower fornix on contact with the ocular surface. The paper absorbs tears. The length of paper wetted over a set of time of 5 minutes is an indication of tear volume $(1995)^{15}$. Normal wetting is more than $15 \mathrm{~mm}$ Reading less than $5 \mathrm{~mm}$. Indicates severe Kerato Conjunctivitis SICCA. Tear film break up time is done to know the stability of tear film and requires observing, the cornea using a slit lamp bio microscope with a broad, beam cobalt-blue light source set at $10 \mathrm{x}$ magnification (1985) ${ }^{14}$, dye impregnated paper strip is placed on the bulbar cornea for a brief moment ask the patient to blink and then ask the patient to refrain from blinking. The time elapsing between a complete blink and the appearance of the $1^{\text {st }}$ dark spot or stroke is measured and taken as break up time. 3 valves are taken and mean value is calculated. The value below 10 seconds is considered abnormal. In this study 44 patients showed dry eyes and is the most common manifestation.

The commonly prescribed treatment modalities were tear substitute (preservative free) gels and emollients for majority of the patients only in 5 patients punctual occlusion by plugs and cauterisation were needed. Tarsorrhaphy was needed in one case only.

Episcleritis had noted in 26 patients (52\%) out of 198. A study by Watson and Hayreh (1976) ${ }^{16}$ on a 10 years study had revealed as 207 episcleritis and 159 scleritis patients. In this study also the number of episcleritis is more than scleritis. The simple diffuse type was commonly found. Topical steroid and NSAID as well as oral NSAIDS are advocated to this patients. Initial treatment should be focused on relieving discomfort and stoppage of progression of the disease.
Scleritis is noted in 12 patients. Mostly of the diffuse anterior type.

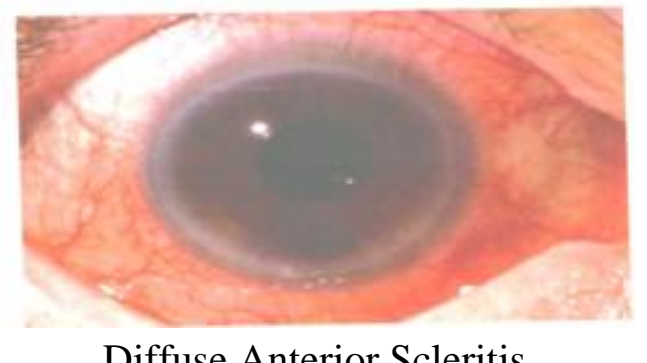

Nodular types are noted 3 pers ons. Nodular scleritis tends to be intermediate in severity between diffuse Anterior Scleritis and necrotizing scleritis with respect to signs and symptoms of disease and final vision outcome.

There is more pain than episcleritis and also there is tenderness on palpation (1998), (1976) ${ }^{18,19}$. Hence the need of therapy is to alleviate the pain and to prevent structural damage to the eye. Oral NSAIDS and steroid drops like (Prednisolone) and systemic corticosteroids are needed in patients with moderate to severe scleritis and slowly tapered to better tolerance dose. Sub conjunctival injection of corticosteroids delivery also given in '4' cases along with topical steroids and cycloplegic and immuno suppressants like cyclosporine drops $(0.05 \%)$. Immuno suppressive drops like cyclosporine which act at least in part by interfering with interleukin-2 has been used with some success in the treatment of scleritis ${ }^{20}$ (1989). Patient with non-necrotising simple diffuse scleritis treated with oral steroids/topical steroids. Non-response with above therapy in 3 cases responds well to immuno suppressants therapy.

PUK was noted in five persons only.

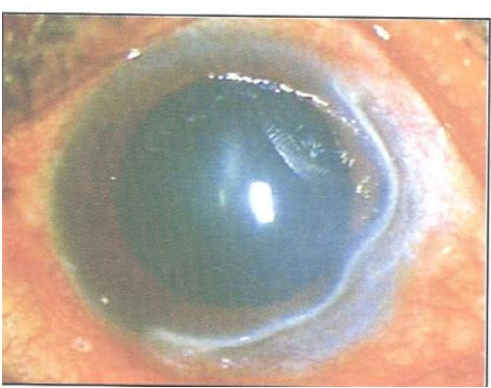

PUK 
1. PUK noted may leads to rapid corneal Keralolysis, perforation of the globe and vision failure and is associated with systemic vasculitis in more than $50 \%$ of cases $(2002)^{22}$. It is a potentially divasting disorder with crescent shaped inflammation at the margin. Treatment modalities were included as topical cycloplegic, $1 \%$ medroxyprogesterone, lubricants and gels with systemic NSAIDS. I.V. (Intra venous)Cortico Steroids were also recommended in selected cases. Amniotic membrane patching done in 2 cases following the procedure less progression of the lesion and stromal Ulceration was noted post operative broad spectrum antibiotics were used for 1-2 weeks along with topical steroids for 6-8 weeks in tapering doses to reduce inflammation systemic immuno suppressants is not required ${ }^{23}$ (2005). Anterior uveilis was noted in 7 patients but no band shaped Keratopathy was noted similar to a study done by P.G. Department of Ophthal, Chettinad Hospitals and Research Institute Chennai, T.N., South India. In 2015 for a one year study revealed 5 (20\%) out of 50 patients with RA showed anterior uveitis without band Keratopathy $(2015)^{6}$. Anterior uveitis is a sight threatening condition with long term sequalae, if not diagnosed and treated early. Hence Anterior uveitis diagnosed patients were treated with proper medications and regular follow up visits also recommended.

Many patients were send for plaquenil (HCQ) toxicity ie many are using it for more than 6 years without any follow up or ophthalmologic evaluation.

Murmor et al (2011) was mentioned that substance such as chloroquine and plaquenil (HCQ) used in the treatment of RA may cause toxic maculopathy with reduced vision actuity and 'bulls eye' in the retina associated with the long duration of treatment. ( $>7$ yrs administration) and the drug dose mean daily dose to minimize toxicity is $6.5 \mathrm{mg} / \mathrm{kgr}$ body mass for HCQ and $5 \mathrm{mg} / \mathrm{kgrm}$ body mass to chloroquine $(2011)^{25}$.

Plaquenil (HCQ) was used in 117 patients 12 out of 117 patients were presented with bulls eye maculopathy on fundus evaluation and lower corneal deposits (Cornea verticillata) was seen in 4 patients only.

Scotoma revealed on amslers grid test to all 12 patients.Instructions like the regular follow up visits to ophthalmology clinic as well as Rheumatology clinic for seeking expert opinion upon any change in the treatment schedule of systems drugs was given. Steroid toxicity in the form of posterior sub-capsular cataract (opacity) was seen in '9' patients. A study conducted by Reddy CD etal at PGI CHANDIGARH, India on 100 patients of RA also revealed a relation and duration of steroid intake and formation of posterior sub-capsular opacity (Cataract) Duration noted as 5 months to 12 years $(1977)^{27}$.

Out of 167 patient's on steroids, 9 showed PSCC. The presence of posterior sub-capsular opacity is noted more in patients who were on long term steroid therapy and there is absence of posterior sub capsular opacity in those who were not taking steroid. This shows the relation between steroid intake and presence of posterior sub capsular opacity the observation of posterior sub capsular opacity in patients with RA who were on long term oral steroids has been well documented by O.Glestly and Williamson ${ }^{\text {etal }} 26$. In the present study the patients with the PSCC were presented with complaints of decreased Vn (Vision) glare and decreased sensitivity to contrast. Vn acuity screening tests and slit lamp evaluation for grading of the cataract was noticed. Current ocular status due to toxicity was explained to them. 4 Patients out of 9 had undergone for cataract surgery. Instructions like follow up visits on regular intervals to those patients who had undergone or not undergone for cataract surgeries were given. 


\section{Conclusion}

High incidences of ophthalmic involvements are noted in this study. Therefore it is essential to seek regular Ophthalmic evaluations as a routine to detect ocular involvements early even though they are asymptomatic to avoid further sight threatening conditions. Among the opthal complications dry eyes is the commonest followed by episcleritis, scleritis, PUK and Iridocyclitis (anterior uveitis) Dry eyes is directly related to the duration of the disease than the severity Routine evaluation tests help to detect the early onset of ophthalmic manifestation and to start treatment. If progressed without treatment may lead to severe vision drop due to corneal scleral involvement or due to ophthalmic complications associated with systemic therapy are evident in this study. Drugs related to this are plaquenil (HCQ) and corticosteroids.

\section{Reference}

1. ALAN J Silman and Jacqueline E Pea soon Epidemiology and Genetics of RA : Arthritis Res.2002, 4,S.265-S 272.

2. PATEL S J and Lundy D.C. (2002) Ocular Manifestation of Auto - Immune Disease 66.

3. Fuerst.D.J., TANZER D J and Smith R.E. (1998), Rheumatoid disease International Opthal Clinics, 38, 47-80. http:idx_doi.org/10. 1097/0000 $4397 \quad$ 199803840-00007.

4. Gordo DA, Stein J L Brodei E The extra articular features of R A AMJ Med/54 : 445, (1973)

5. Lille by V, Gran J T., Tidsskr Nor Laege Foren 1997 ; 117 : 4223-5.

6. Charanyac, Swathi A., Jantis Pandurangan.R., Ocular Manifestation IN RA Patients presenting IN Tertiary Care Hospital in South India A prospective study Int. JSCI Stud 2015 ; 3 (8) 61-66).

7. Wanchu.A., Suni L, Deodhar S D et al Extra Articular Manifestation among North India patients RA Journal of Indian Rheumatism
Association 1997 ; 5 (3) 75-8.

8. Reddy SC, Gupta S.D Jain I.S Deodhar S D Ocular Manifestations of Rheumatoid Arthritis Indian J Ophthalmol 197725 : 206.

9. Moss S E, K lein R., Klein B E, Prevalence of and risk factors for dry eye syndrome Arch Opthalmol 2000118 : 126-4.

10. Felson D T AND ERSON J J, Boers M Bombardier C, Furst D, Goldsmith C et al. American College of Rheumatology, Preliminary definition of improvement IN Rheumatoid Arthritis Rheum 1995 ; 38, 727-35.

11. S.Kalke. S D Perera, N D Patel, T.E.Gordar, B.Das Gupta Rheumatoid volume 40, Issue 2, Feb.2001, Pages 170-177.

12. Toda 1 Fujishuma H, T Subotak, Ocular Fatique is the major component of dry eye Acta. Ophthalmol 71; 347-352, 1993.

13. Shizato.M Yamamoto J Hirata C.E Goldbey CA, Yoschinari N.H and Bonfa.E., (2008) Ege disease in a patient with Rheumatoid arthritis Vogt - Koyanagi Harady Syndrome and Rheumatoid Arthritis.

14. Meghner L S., Bron A.J., Tongu S.R., Gilbert D.J effect of fluorescen. Instillation on the pre-corneal tear film stability curr eye Res. 4: 9-12 1985.

15. Bandem - Roche $\mathrm{K}$ et al : Challenges to defining and quantifying dry eye. Invest Ophthalmol Vis. Sci. 36 : S 862, 1995.

16. Watson PG, Hayreh S S, Scleritis and Episcleritis Br.J Ophthalmol 1976 : 60 : 163-91.

17. MC GAVIN DD, Willianson J, Forrester J V., Foulds W.S., Buchanan W W, Dick W C et al Episcleritis and scleritis. A study on their clinical manifestation and assosication with Rheumatoid Arthritis Br. J Opthalmol 1976 : 60 - 192-226.

18. Harper.S.L., Foster.C.S., (1998) The Ocular Manifestation of R.A. International Opthalmology Clinics, 38, 1-19. http://dx.doi./org/ 10. 1097/00004397 - 
$199803810-00003$.

19. MC GAVIN D D William Son.J, Forrester.J.V., Foulds, W.S Buchanan W.W.Dick W.C. et al; (1976) Episcleritis and Scleritis. A study on their Clinical Manifestation and association with Rheumatoid Arthritis. British Journal of Ophthalmology 60: 192 - 226. http// dx.doi.org/10.1136/610.60. 3:192.

20. Wake field D, MC closky.P. Cyclosporin therapy for severe scleriotis. Br.J. Ophthalmol/ 1989:73 743-6.

21. RAO NA, FONTRL. Pseudo Rheumatoid Nodule of the Ocular Adenexa. AMJ Ophthalmol $70: 471$.

22. Sule.A, Balakrishnan.C., Gaitonde.S., Mittal.G., Pathan E, Gokhale.N.S/ and Joshi V.R. (2002) Rheumatoid Corneal melt. Rheumatology 41-705-706. http:/dx:doiOrg./10. 1093/ Rheumatology/41.6.705.

23. Kobayashi.A, Ijiris, Sugiyamak D Pasculae M.A. Tseng SC. Detection of Corneal Epithelial defect through amniotic membrane patch by Fluorescein : Cornea 2005. 24:359-60.

24. Del Castillo, B.J.M., Diaz - Valle D, Pato, E. Abad.L.C. and Ale jandre.N (2008). Articular disease and Uveitis Anales del sislema Sanitario de Navarra.31.

25. Marmor.M.F., Kellner U., Lai. T.Y., Lyons.J.S. and Mieler, w.f. (2011). American Academy of Ophthalmology Revised Recommendation on screening for chloroquine and Hydroxy Chloroquine, Retinopathy.

26. Oglesby R.B., Black.R.L., Saltman L Ven and Bunin.J.J 196/Arch Ophth. 66.519.

27. Reddy SC, Gupta.S.D., Jain.I.S., Deodhar.S.D., Ocular Manifestation of RA Indian J Ophthalmol 1977, 25 : 20-6.

28. Thompson M, Eadie.S Kerato Conjunctivities SICCA and Rheumatoid Arthritis ANN Rheum Dis. 1956 : 15:21. 